\title{
Introducción de actividades de Gamificación en una asignatura humanista para informáticos
}

Juan Vte. Oltra Gutiérrez ${ }^{\mathrm{a}}$, Fernando J. Garrigos-Simón ${ }^{\mathrm{b}}$ y Yeamduan Narangajavana ${ }^{c}$

${ }^{a}$ UniversitatPolitècnica de València, jvoltra@omp.upv.es, ${ }^{b}$ UniversitatPolitècnica de València ${ }^{c}$ Universitat Jaume I

\begin{abstract}
It is in this communication to present an experience of "gamification" held in during the 2015-2016 course at the Escuela Técnica Superior de Ingeniería Informática de la Universitat Politècnica de València, (here in after ETSINF$U P V)$, where iten courages students compete through three possible channels: Twitter and both face discussions in classroom and online mode (throughforums)

The activities of "gamification" carriedout in the Deontologia $y$ Profesionalismo, 2nd course of ETSINF-UPV and the questionnaire will assess your application is presented relate.
\end{abstract}

Keywords: Gamification, game, experience, competition, training

\begin{abstract}
Resumen
Se trata en ésta comunicación de exponer una experiencia de "gamificación", realizada en durante el curso 2015-2016 en la Escuela Técnica Superior de Ingeniería Informática de la Universitat Politècnica de València, (en adelante ETSINF-UPV), donde se alienta a los alumnos a competir mediante tres vías posibles: Twitter y debates tanto presenciales en aula como de modo online (mediante foros)

Se relacionan las actividades de "gamificación" llevadas a cabo en la asignatura Deontología y Profesionalismo, de $2^{\circ}$ curso de la ETSINF-UPV y se presenta el cuestionario que permitirá evaluar su aplicación.
\end{abstract}

Palabras clave:Gamificación, juego, experiencia, competición, formación

\section{Introducción}

La asignatura donde se ha desarrollado la experiencia, Deontología y Profesionalismo, tiene unos cuantos elementos que la hacen singular entre sus compañeras de la ETSINF-UPV.

Por una parte, en los grupos donde se ha desarrollado la experiencia, ésta convivía con un modelo de docencia alejado de los parámetros clásicos, pues se aplicaba en ellos FLIPTeaching (Puede verse una descripción del diseño de la experiencia en (OLTRA, 2015)). 
Además, la asignatura tiene un corte marcadamente humanista, pues tanto al hablar del marco regulatorio de la profesión, donde se pone especial acento en elementos jurídicos, como de su marco deontológico, espacio del temario donde esta característica se hace aún más evidente, provoca en el alumno, acostumbrado a recibir docencia sobre programación de semiconductores, algorítmica u otras batallas tecnológicas de similar calado, un cierto estado de perplejidad (dicho en román paladino: ¿Quién ese tipo tan feo y de qué me está hablando?)

Singularmente, este hecho diferencial ha provocado, desde el origen de la asignatura, repetidos intentos de localizar la llave que permita abrir la caja del interés en el alumnado. Acercarle a la teoría mediante el modelo "FlipTeaching" ha sido una de las claves que, al permitir disponer de buena parte del tiempo de aula habitualmente destinado a la típica clase magistral, dar sentido práctico en el subconsciente colectivo del alumnado presente mediante casos a la materia. Con la "gamificación" se establece una sana competencia que apoya ese afán.

\section{Conceptos}

Usamos el término, poco apropiado dentro de una ortodoxa aplicación de la lengua española, de "gamificar" por su extenso uso dentro de la comunidad docente. Y, dado que no parece perturbar al idioma, lo usaremos en adelante, con sus derivados, sin las comillas que lo encierran. Descartamos así otros términos, a buen seguro más correctos desde un punto de vista lingüístico, pero con menos ecos en nuestra profesión, como ludificación, jueguización o juguetización ${ }^{1}$.

Queda definirlo, para lo que recurrimos a (FUCHS, 2014), quien nos da un doble posible enfoque: en primer lugar, como un proceso general en el que los juegos y experiencias lúdicas se entienden como componentes esenciales de la sociedad y la cultura. Desde esta perspectiva podríamos considerar cómo las prácticas y rituales, pertenecientes a diferentes contextos históricos y culturales, podría tomar la forma de un juego. Desde esta perspectiva, puede ser visto como un fenómeno amplio, no tan novedoso como muchos nos quieren hacer creer. El segundo enfoque es el que nos es más cercano, definiéndolo como una práctica mucho más limitada. Este segundo significado, mucho más extendido, se ha usado por parte de expertos en marketing, del diseño... y de la docencia, entre otros. Siempre siguiendo a Fuchs, apostillamos que su primer uso documentado data de 2008, siendo adoptado de manera general en la segunda mitad de 2010.

Podemos entonces centrarnos en esta segunda acepción, siendo pues para nosotros el uso de, elementos y dinámicas propias de los juegos en actividades no lúdicas con fines distintos que van del apoyo al aprendizaje, potenciar la motivación o solucionar problemas.

\footnotetext{
${ }^{1}$ Escúchese a tal fin a D. Fernando LázaroCarreter, quienfuera director de la Real Academia Española, ensuconferenciapronunciadaen la fundación March el 2 de febrero de 1982: "Comunicaciónpoética y comunicaciónpráctica". (CARRETER, 1982)
}

(cc) EY-NC-ND 2016, UniversitatPolitècnica de València 
En este sentido es en el que lo usamos, intentando aplicar esquemas y estructuras de los juegos en una actividad que no está a priori diseñada para seguirlos ni, mucho menos, divertirse: la formación. El elemento estrella de este tipo de actividades suele ser los premios, las pequeñas recompensas una vez se alcanzan pequeños logros. Y en esto, no ha sido una excepción la presente experiencia.

Efectivamente, se van presentando al alumnado pequeñas actividades, de carácter voluntario, incitándoles a la participación $\mathrm{y}$, como veremos, estableciendo cierta competición entre ellos. Una vez finalizada cada actividad, se premian las mejores intervenciones $\mathrm{y}$, de forma anónima para preservar la privacidad, pero de forma que cada uno pueda identificarse claramente, se indican los resultados para que un alumno concreto pueda conocer su "situación en el ranking". Esto permite convertir puntuación en premio de forma directa, otorgando 0,1 punto a cada vencedor en cada actividad. Un valor casi despreciable en el global de la asignatura, que pero que ofrece a los participantes un aliciente difícil de obviar al ver convertirse directamente sus esfuerzos en un pequeño pellizquito de nota extra para su expediente final.

Resulta obvio, pero cabe en este momento hacer una precisión: la gamificación no descansa en la premisa de convertirlo a todo en un juego, sino en usar lo mejor de ellos: en un juego la gente participa una y otra vez aunque pierda, tiene su punto divertido y, para los propósitos que se plantean en la asignatura (que el alumno sepa más, aprenda a razonar adaptando los conocimientos que se le dan y busque por su cuenta otros nuevos, actualizaciones o adicionales a los dados por el docente) nos encajan, usando la típica expresión castellana, como un guante.

Para ello, descomponemos los elementos definitorios de un juego típico, haciendo una adaptación muy particular de (MATERA, 2015). Así, tenemos que hay unas reglas que se han de seguir, un sistema de retroalimentación y la voluntariedad. Estas tres particularidades son las que nos han encaminado.

Los beneficios que hemos esbozado que se pretenden obtener, van orlados de una intención previa que, ojalá, se alcance: aportar un mayor atractivo y motivación para nuestra materia. De partida, redactando estas líneas a finales del mes de marzo, con la asignatura llegando a su ecuador (es de segundo semestre) si se puede anticipar que el compromiso de los alumnos que han decidido "jugar" (de momento, alrededor de un 25\% de los matriculados) se mantiene firme y que entre ellos se ha establecido algo muy típico que se suele observar en los jugadores online de videojuegos: cierto reconocimiento entre sí de los logros alcanzados.

Hay diversas mecánicas a aplicar en los juegos, desde asignar valores cuantitativos a determinadas acciones (puntuar) a dar regalos. Para esta experiencia se ha desestimado ofrecer "regalo" alguno, no solo premios extra, sino incluso bienes virtuales. La opción ha sido puntuar, estableciendo clasificaciones que permitan a los participantes compararse entre sí y marcando un umbral, a partir del cual pasan a un nivel superior. Volveremos a esto cuando se detallen las estrategias. Se ha desestimado establecer desafíos competitivos, 
más allá de obtener una mayor puntuación. Por ello tampoco se han establecido "misiones", entendiendo como tales las consecuciones de desafíos concretos planteados.

Para el desarrollo de la presente experiencia se han puesto en marcha tres vías. Al final de cada semana de clases, se evalúan las participaciones de los alumnos en cada una de ellas y se puntúan, pudiendo una actividad tener un "vencedor", varios (ex aequo) o ninguno (declararse desierta), resultados que se dan a conocer inmediatamente. Cíclicamente se publican clasificaciones que permiten a los participantes ver cómo están situados en "su camino al pódium".

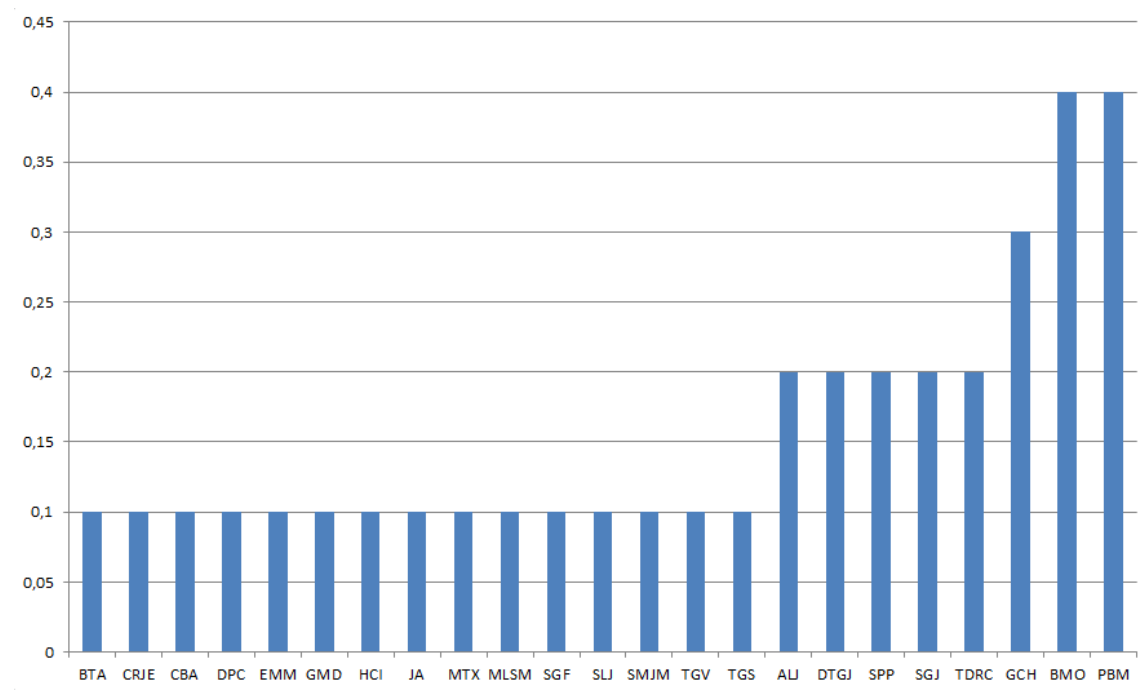

Fig. 1 Ejemplo de clasificación mostrada a los alumnos. Elaboración propia

\section{Objetivos}

Como se ha apuntado, se usan tres vías para la gamificación, que detallaremos a continuación. El principal objetivo es determinar cuál es la mejor de esas vías, con idea de poder, en cursos futuros, apostar más firmemente por ella.

Dado que en el momento de redactar la presente comunicación la actividad está en curso, tan solo trazaremos el mecanismo para poder realizar esa selección.

Para ello, se ha previsto medir la participación midiéndola temporalmente en tres bloques, cada uno de cinco semanas docentes (la asignatura tiene quince sesiones de teoría), revisando en particular, además del número total de alumnos en cada una de las tres posibilidades, como se han ido sumando (o abandonando) cada una de ellas.

Para comprobar su efectividad, estableceremos si es posible relaciones entre los resultados académicos obtenidos por los alumnos que han participado con el resto $\mathrm{y}$, en particular, con su "tanteo" alcanzado en la gamificación con los resultados finales en la asignatura.

Por último, se ha desarrollado una breve encuesta, con el propósito de que nos permita acercarnos a la percepción del alumno sobre la experiencia.

(cc) EY-NC-ND 2016, UniversitatPolitècnica de València 


\section{Desarrollo de la innovación}

Como se ha anticipado, se han empleado tres vías para la gamificación, como distintas estrategias de acercamiento.

Estas son, sin ánimo de dar una definición exhaustiva de cada una de ellas, las siguientes:

- Uso de Twitter. Desde la cuenta del profesor, se mantiene un contacto con los alumnos, evidentemente voluntario por su parte (hay quien quiere seguir a la cuenta, y quien no). Dentro de esos contactos, hay una serie de tuits que, acompañados del hastag \#DyP que los identifica como relativos a la asignatura, contienen noticias o comentarios relativos a temas vistos o próximamente a ver en las sesiones de la asignatura. Se "premia" el mejor comentario que bien pude ser simplemente un texto aclaratoria, un enlace que mejore la percepción de lo expuesto e, incluso, un desmentido si la noticia originalmente comentada resulta ser falsa. Estas actividades en concreto se han desarrollado en el marco del PIME "INTEGRACION DE RECURSOS TECNOLOGICOS EN LA ENSEÑANZA: USO DE TWITTER Y FACEBOOK EN LA DOCENCIA UNIVERSITARIA"que acoje esta experiencia.

- Uso de los foros de la asignatura. Usando las herramientas que nos ofrece la plataforma Poliformat de la Universitat Politécnica de Valencia, se generan debates online sobre casos vistos en la asignatura. Su duración es de una semana. Al cabo de la misma, las mejores participaciones por relevancia, por contenidos apropiados y, también, por saber mover a la participación provocando respuestas, son premiadas.

- Debates en aula. Algunas de las sesiones presenciales terminan con un debate de aula sobre un caso real que se analiza a la luz de los contenidos teóricos de la asignatura. La única forma en que se anima a la participación en esos debates es "gamificándolos". Para ello, el profesor va provisto de una suerte de billetes de juguete (ver imagen) y, cuando un alumno interviene con una exposición que es brillante, por dar justo en el clavo cuando el asunto es complejo, o por descubrir una derivada del debate que es interesante, se le da uno. Al final de la clase, los alumnos con premio "pasan por caja" y el profesor les apunta la calificación, siendo éste el único caso de los tres donde el alumno conoce inmediatamente su resultado de participar en la actividad. 


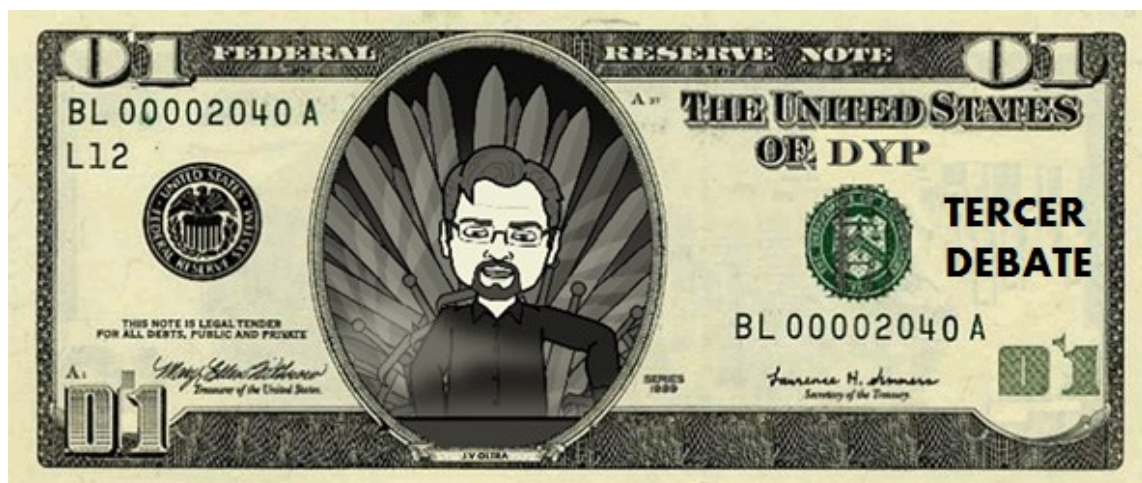

Fig.2Ejemplo de billete usado en los debates. Elaboración propia

Citábamos también en el apartado de conceptos el umbral. Éste se ha situado en 0,5 puntos. Cuando un alumno lo supera, el profesor le felicita en el aula, siendo ésta felicitación un tipo de recompensa diferente, donde no se convierte directamente en puntos pero que anima al estudiante a seguir participando. Hay que coincidir con Matera (MATERA, 2015) en que el aprendizaje basado en la gamificación se aprovecha de la imaginación y la ambición.

Siguiendo a manera, y considerando que se trata de una materia donde el número de participantes podía ser muy elevado, se ha optado por buscar, en lo cualitativo, la cooperación de los estudiantes (que muchas veces se apoyan unos en otros en sus comentarios) y, resulta obvio decirlo, en la competición. Puede verse de forma gráfica en la adaptación que hemos hecho del texto de Matera en la imagen siguiente: 


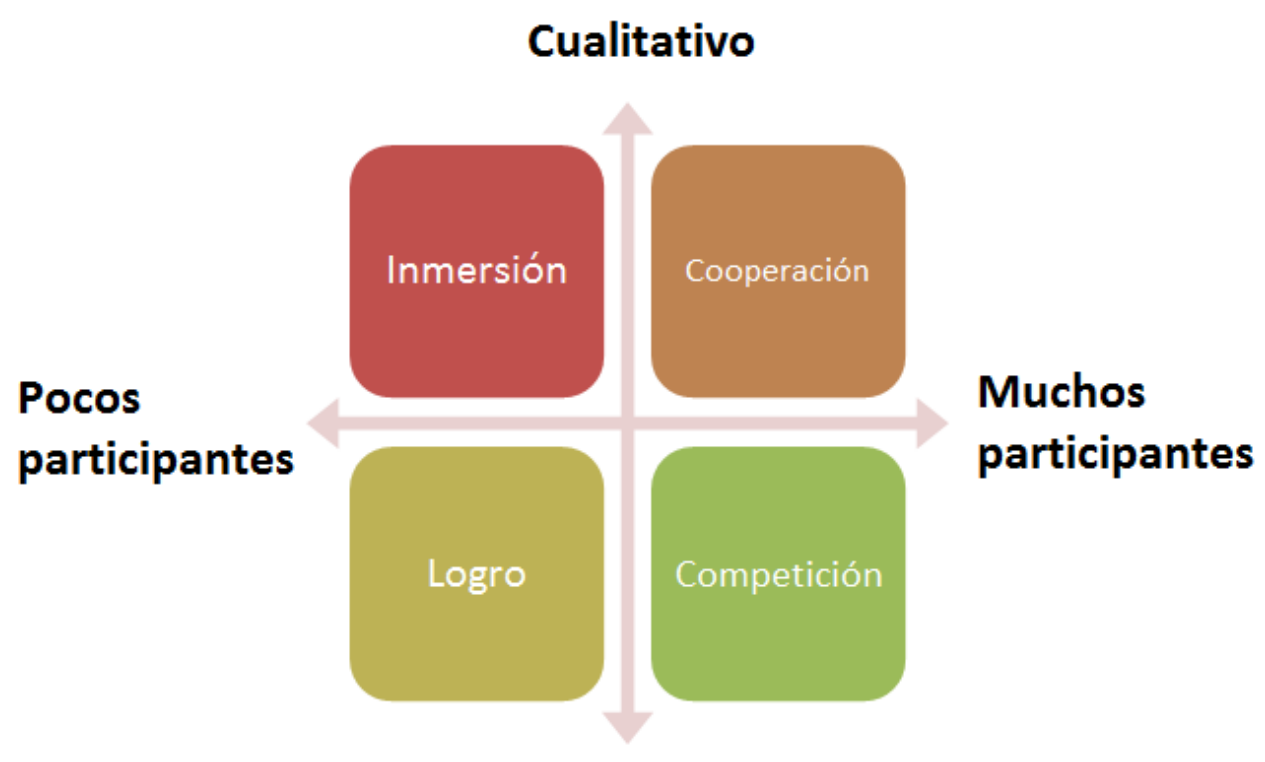

\section{Cuantitativo}

Fig.3. Objetivos de la gamificación, adaptado de (MATERA, 2015)

\section{Resultados}

Aun con la asignatura en desarrollo, lejos de su ecuador docente, podemos hablar de pocos datos cuantitativos, tan solo podemos hablar de lo cualitativo, y eso conlleva el peligro de toda percepción subjetiva.

Con una participación de un 30\% de los matriculados en alguna de las vías de participación, y de un $20 \%$ de forma regular, podemos ver que muchos de ellos tienen más interés en conocer porque no han acertado que en su éxito concreto en una u otra actividad. Es algo que corrobora a Matera, cuando nos dice que "Tenemos que ayudar a los estudiantes a entender que ellos deben aprender tanto, si no más, de sus fracasos como de sus éxitos" (MATERA, 2015). Aún más: algunos de ellos, con la actividad terminada y ya puntuada, han solicitado información adicional, haciéndonos regresar una vez más a Matera (MATERA, 2015) para decir con él que "debemos inspirarles a elevarse por encima de la mediocridad y hacer lo extraordinario" (MATERA, 2015)

En algún momento, y de forma accidental, se ha detectado que alguno de los participantes, que se ha visto ya subido en ese pódium virtual de la excelencia que supone el umbral que citábamos, "regalaba" enlaces de interés y ayudas a otros compañeros, reviviendo esa sombra del Potlach que Fuchs introduce en su brillante texto. (FUCHS, 2014) y encajando perfectamente en el ámbito afectivo de la taxonomía de Bloom (BLOOM, 1981) 
Queda pendiente, para la última semana de mayo, pasar una encuesta, con las preguntas que pueden verse en la siguiente imagen.

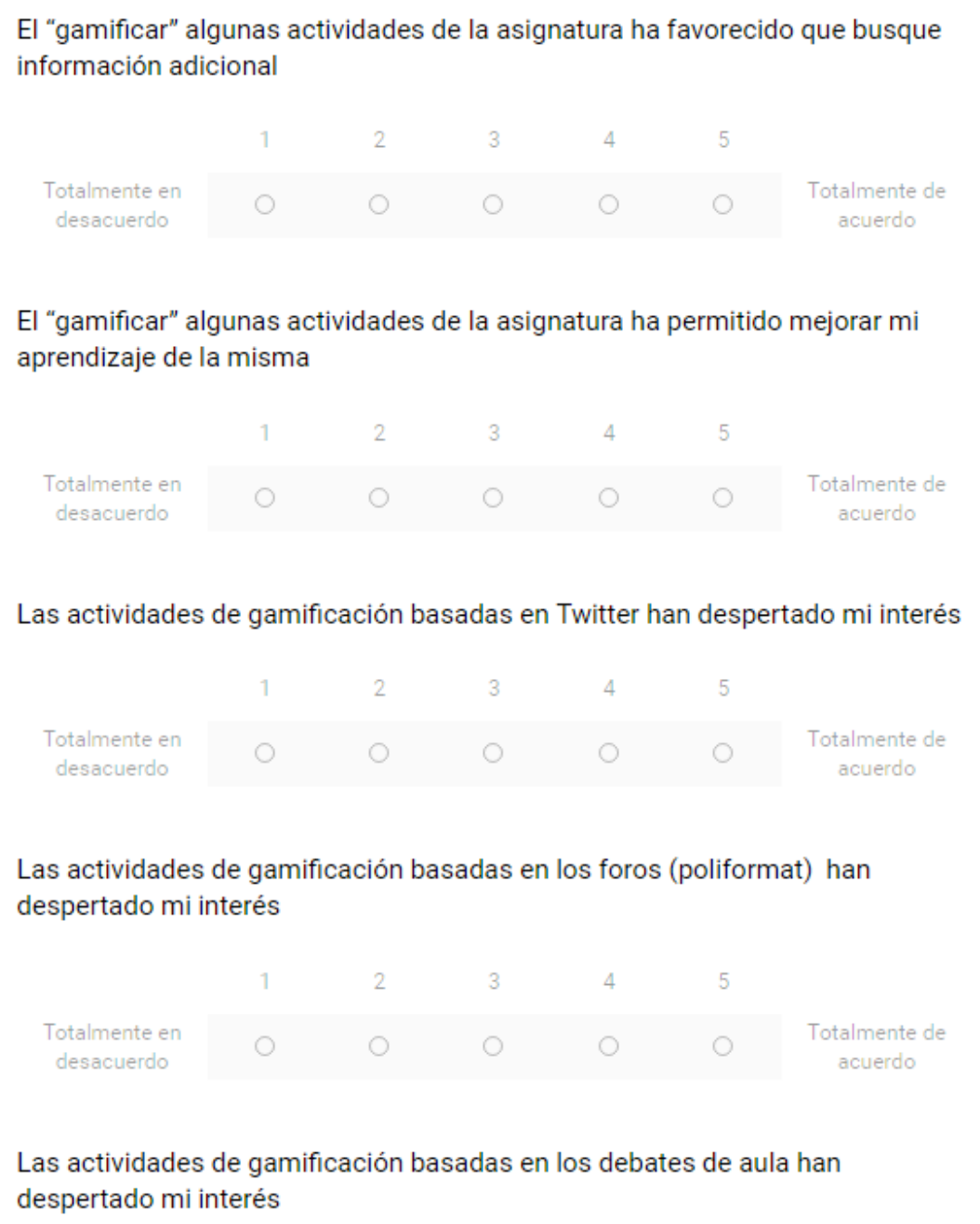

Fig.4Encuesta para losestudiantes, elaboraciónpropia

Somos conscientes de la parquedad de los resultados cuantitativos aportados, algo de dificil solución hasta obtener los resultados de la encuesta mencionada. Los únicos que se pueden aportar son de caracter cualitativo, y su misma enumeración, dado que proceden de las percepciones del profesorado, sin contrastar por la encuesta, carecerían de rigor científico alguno.

No se analizan en paralelo experiencias similares pues, en un contexto similar (asignatura perteneciente a la educación superior, con las características singulares que enunciamos al principio), no hemos localizado. De cara al próximo curso, tenemos noticia de que al menos un par de asignaturas tienen intención de aplicar esta misma experiencia bajo los mismos parámetros, pudiendo entonces ser analizadas de forma comparativa. Una de esas 
asignaturas se situa en el master MUGI (Master Universitario en Gestión de la Información) y otra en el grado de derecho.

\section{Conclusiones}

Cabe considerar, más en la asignatura en la que se aplica la gamificación, que descansa buena parte de su temario en la deontología, la ética, si estas estrategias son correctas.

Siguiendo a (FUCHS, 2014) vemos como casi desde el primer día, este tipo de actividades han sido criticadas como manipuladoras, explotadoras, o coercitivas. La respuesta que da a estas críticas es fulminante y la tomo como propia: si consideráramos stricto sensu no solo la gamificación, sino cualquier actividad en aula, resultaría que no podríamos ni comunicarnos con el alumnado por el riesgo implícito de influir en ellos: cualquier comunicación y acción afecta a los demás. Cada actuación abre debates sobre la ética deliberación y la consecuente toma de decisiones. Así, consideramos que la gamificación no es inherentemente "más" o "menos" ética. La gamificación encajaría en lo que el autor llama "diseño persuasivo" que no tiene por qué ser incorrecto si las actuaciones individuales son éticas en sí mismas, no producen efectos negativos para los alumnos y son suficientemente informadas.

En el caso particular de esta asignatura, con los pocos datos que podemos barajar dado que aún se encuentra en curso, las previsiones son muy optimistas. De entrada podemos decir que hemos superado el filtro que establece Burke (BURKE, 2014) (de forma gráfica, en la imagen siguiente) equilibrando motivación, recompensa y entretenimiento. Acaso podamos decir, a fin de todo, que hemos hecho bueno el viejo adagio que nos impelía a enseñar deleitando.

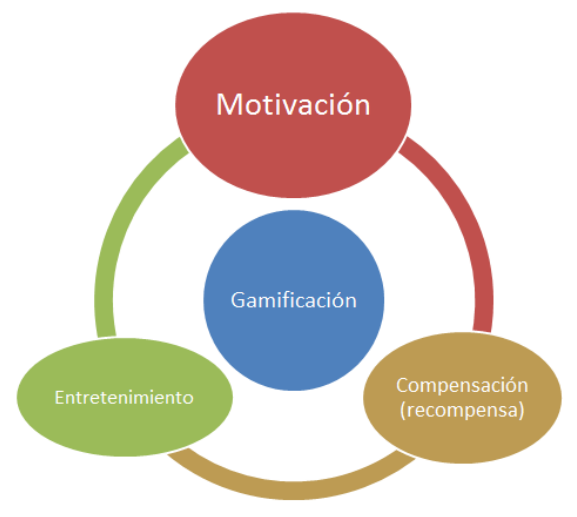

\section{Referencias}

BLOOM, B. S. (1981) Taxonomia de losobjetivos de la educación. Buenos Aires: Libreria de Ateneo

(cc) EY-NC-ND 2016, UniversitatPolitècnica de València

Congreso In-Red (2016) 
BURKE B. (2014) Gamify: how gamification motivates people to do extraordinary things Massachusetts: Bibliomotion, Inc.

FUCHS, M., FIZEK, S., RUFFINO P., SCHRAPE, N. (2014) Rethinking Gamification. Lüneburg, Alemania:Meson press.

LÁZARO CARRETER, F. (1982) “Comunicación poética y comunicación práctica". Fundación March. Disponible en<http://www.march.es/conferencias/anteriores/voz.aspx?p1=2312\&l=1\&utm_medium=b uscador\&utm_campaign=tematicos\&utm_source $=$ fjm $1 \% \mathrm{C} 3 \% \mathrm{~A} 1 \mathrm{zaro} \% 20$ carreter $>$

[Consulta: 3 de marzo de 2016]

MATERA, MICHAEL (2015) Explore Like a Pirate. San Diego, CA: Dave Burgess Consulting, Inc.

OLTRA GUTIÉRREZ, J.V. (2015). “Diseño de unaexperiencia de Flip-Teaching para la asignaturaDeontología y Profesionalismoaimpartiren la EscuelaTécnica Superior de IngenieraInformática de la UPV". EnCongreso Nacional de InnovaciónEducativa y de Docenciaen Red. Valencia. Disponibleen $:<$ https://riunet.upv.es/handle/10251/52755 $>$ [Consulta: 3 de marzo de 2016]

AUTOR O INSTITUCIÓN (@usuario). “Contenidodeltuit”. Fecha, horadelmensaje, [Twitter]. $<$ URL $>$ [Consulta: fecha]. 